$\xi=$

\title{
Comparison between bicarbonate-based and Stewart methods to assess metabolic acid-base disorders in internistic patients. A pilot study
}

\author{
Valenti Maria 1, Bruno Francesca 2, Demma Shirin 1, Bruno Cosimo Marcello*1 \\ ${ }^{1}$ Department of Clinical and Experimental Medicine, section of Internal and Emergency Medicine, University of Catania, Catania.Italy \\ ${ }^{2}$ Department of Clinical and Experimental Medicine, section of Nephrology, University of Catania, Catania. Italy \\ *Corresponding author E-mail: cmbruno@unict.it
}

\begin{abstract}
Aim: To assess the acid-base status in a cohort of internistic patients, using traditional and "modern" methods, to compare their different sensitivity to detect metabolic disorders and to evaluate a possible relationship between classical and alternative parameters.

Patients and Methods: 143 assessment of acid-base and electrolytes balance on 121 internistic patients (76 males and 45 females, mean age $73.9 \pm 10,8$ years) were examined according to bicarbonate-based and Stewart methods.

Results: The traditional method detected 81 cases $(56.6 \%)$ of metabolic alkalosis and 15 cases $(10.4 \%)$ of metabolic acidosis. The Stewart method detected 92 cases $(64.3 \%)$ of metabolic alkalosis and 22 cases (15.3\%) of metabolic acidosis.

Traditional method failed to detect 11 cases of metabolic alkalosis (chi square $=1.443 ; \mathrm{p}=0.226$ ), and 7 cases of metabolic acidosis (chi square $=1.118 ; p=0.290$ ) when compared to Stewart's method. A significant relationship was observed between Strong Ion Gap (SIG) and Anion Gap corrected for albumin concentration (AGcorr) $(r=0.53 ; p<0.001)$.

Conclusions: Our result showed that traditional method is useful to assess acid-base status in internistic clinical setting as well as Stewart's method because no significant difference was found between the two approaches. Nevertheless, the light disagreement observed between the two methods suggests that in a small percentage of cases the traditional method can fail to detect metabolic acid-base abnormalities.
\end{abstract}

Keywords: Acid-Base Disorders; Stewart Disorders; Stewart Method; Metabolic Acidosis; Metabolic Alkalosis.

\section{Introduction}

Although acid-base disorders are frequently encountered in medical practice, especially in critical patients, the methodology for their approach and interpretation is still being of debate, particularly with regard to metabolic disorders.

Traditionally, diagnostic approach to metabolic acid-base balance is based on bicarbonate-centered theory (Bronsted 1923, Lowry 1923). Two different methods evolved from this theory: the comparative bicarbonate ions/ carbon dioxide tension ( $-\mathrm{HCO} 3 / \mathrm{pCO} 2)$ approach based upon the Henderson-Hasselbalch equation $(\mathrm{pH}=$ $6.1+\log$-HCO3/0.03 pCO2) and "base excess" approach, based on the Van Slyke equation as developed by Siggard-Anderson formula (Singer et al. 1948, Siggaard-Andersen 1977).

The anion gap (AG) was subsequently introduced as difference between measured cations and measured anions (Emmit and al. 1977). AG has been used to establish if metabolic acidosis is due to gain of acid or loss of bicarbonate (Gabow et al. 1980).

In the past years, clinicians were divided between supporters of comparative approach, advocated by the School of Boston and supporters of "base excess" approach advocated by the School of Copenhagen.

In addition to this well-known trans-Atlantic debate (Severinghaus 1993), Stewart, a Canadian physiologist, at the beginning of the 80 s of the last century, proposed a new approach to acid-base disorders (Stewart 1981, 1983).
Stewart identified three independent variables that affect hydrogen ions $\left(\mathrm{H}^{+}\right)$concentration and consequently $\mathrm{pH}$ :

1) Strong Ion Difference (SID) i.e. the difference in the net charge of fixed cations and anions fully dissociated (sodium, potassium, calcium, magnesium) - (chloride + lactate ); under normal condition, this difference is about $38-42 \mathrm{mEq}$;

2) total weak acid concentration (A-tot) i.e. partially dissociated weak acids; albumin and phosphate are weak acids and their degree of dissociation is related to temperature and $\mathrm{pH}$; under normal condition (albumin $4.4 \mathrm{~g} / \mathrm{dl}$ and inorganic phosphate $3.0-4.5 \mathrm{mg} / \mathrm{dl}$ ) A-tot value is about $15 \mathrm{mmol} / \mathrm{L}$.

3) $\mathrm{pCO} 2$.

Given the constraint of the law of electroneutrality, the net positive charge represented by the SID is balanced by nonvolatile buffers: bicarbonate ions and Atot.

Thus, the difference in the fixed ions is named apparent SID (SIDa) whereas the sum of ${ }^{-} \mathrm{HCO} 3$ and A-tot is named effective SID (SIDe). The difference between SIDa and SIDe is named strong ion gap (SIG) and, under normal conditions, is zero or near zero.

As well as in the traditional method, respiratory disorders are due to a primary variation of $\mathrm{pCO} 2$. Metabolic disorders, according to Stewart approach, are due to a primary variation of independent variables SID and/or A`tot, while hydrogen ion $(\mathrm{H})^{+}$and bicarbonate concentration are considered dependent variables that vary only as a result of the change in one or more independent variables. 
Increase of SID and/ or reduction of A-tot determines a metabolic alkalosis; a reduction of SID and/ or increase of Atot causes metabolic acidosis. An increased SIG value suggests the presence of unmeasured strong anion similarly to anion gap in the traditional method.

The Stewart's approach, also called "modern" or "quantitative," was, initially, very much criticized, but, in recent years, it has been increasing used, especially in intensive care units (ICU).

Actually, literature data are controversial. Some authors (Balasubramanyan et al. 1999, Fencl et al. 2000, Kaplan et al. 2008, Boniatti 2009, Guerin et al. 2010) would prefer the method of Stewart because they consider it more sensitive. Other authors (Sirker et al. 2002, Moviat et al. 2003, Dubin et al. 2007, Kurtz et al. 2008, Rastegar 2009, Masevicius \& Dubin 2015), instead, believe that it would not bring major improvements to traditional methods.

Aim of this preliminary study was to assess the acid-base status in a cohort of internistic patients, using bicarbonate-based and Stewart's methods, to compare their different sensitivity in detecting metabolic disorders and to evaluate a possible relationship between classical and alternative parameters.

\section{Materials and methods}

We carried out a pilot study based on 143 assessments of acidbase and electrolytes balance on 121 consecutive patients (76 males and 45 females, mean age $73.9 \pm 10.8$ years) admitted to Internal Medicine Unit of our Hospital. The study was performed according to Helsinki Declaration. As the blood tests and data collected for this study were all standard clinical practice, written consent was not required and only oral consent was obtained from patients or their kin.

In each patient, blood samples were collected for gas analysis and contemporary determination of serum sodium $\left(\mathrm{Na}^{+}\right)$, potassium $\left(\mathrm{K}^{+}\right)$, chloride $\left(\mathrm{Cl}^{-}\right)$, magnesium $\left(\mathrm{Mg}^{2+}\right)$, inorganic phosphate $(\mathrm{Pi})$ and plasma albumin (Alb).

Arterial blood gas analysis was assayed by IL GEM PREMIER 3000 Analyzer (Instrumentation Laboratory). pH, pCO2, ionized calcium $\left(\mathrm{Ca}^{2+}\right)$ and lactate were directly measured by analyzer and plasma bicarbonate (-HCO3) was calculated by an integrated software from the instrument.

Serum electrolytes, inorganic phosphate and plasma albumin were determined in our hospital laboratory certified EN ISO 9001:2000. Anion gap (AG) was calculated with the simplified formula:

$\mathrm{Na}^{+}-\left(\mathrm{Cl}^{-}+{ }^{-} \mathrm{HCO} 3\right)$ and adjusted (AG corr) for plasma albumin and serum phoshate: $\mathrm{AG}$ corr $=\mathrm{AG}+(0.25 \mathrm{x}[44-\mathrm{alb}(\mathrm{g} / \mathrm{l})]+0.5$ (Pi mg/dl).

In each patient, acid-base status was assayed in agreement to bicarbonate-based and Stewart methods.

According to bicarbonate-based method, $\mathrm{pH}, \mathrm{pCO} 2$ and ${ }^{-} \mathrm{HCO} 3$ were comparatively examined and the rule of expected compensatory response (Rastegar 2005) was applied. AGcorr was also determined to assess the presence of undetected strong anion.

According to Stewart's method, independent variables were calculated: $1, \mathrm{SIDa}=\left[\mathrm{Na}^{+}+\mathrm{K}^{+}+\mathrm{Ca}^{2+}+\mathrm{Mg}^{2+}\right]-\left[\mathrm{Cl}^{-}+\right.$lactate $] ; 2, \mathrm{~A}^{-}$tot $=$ the negative charges primarily contributed by albumin and phosphate in the ionized form in agreement to the formula $\{[\mathrm{Alb}]$ $*(0.123 * \mathrm{pH}-0.631)\}+\{[\mathrm{Pi}] *(0.309 * \mathrm{pH}-0.469)\}$ where albumin concentration is $\mathrm{g} / \mathrm{l}$ and phosphate $\mathrm{mmol} / \mathrm{l}$. Moreover,
SIDe (-HCO3 + A'tot) and SIG (by subtracting SIDe from the SIDa) were calculated. Reference SIDa values were previously obtained from 15 healthy volunteers. Normal range was 37-43 $\mathrm{mEq} / \mathrm{L}$. Upper limit for SIG value was considered $8 \mathrm{mEq} / \mathrm{L}$ according to Fencl (Fencl et al. 2000).

Statistical analysis was performed by chi square test to compare the prevalence of each metabolic disorder in the study series. Linear regression test was used to compare AGcorr and SIG. Statistical significance was set at $\mathrm{p}<0.05$.

\section{Results}

Among 143 determinations, $91(63.6 \%)$ and $98(8.5 \%)$ revealed a metabolic disturbance in agreement to traditional and Stewart approach, respectively.

Metabolic alkalosis was the most common disorder. The traditional method detected 81 cases $(56.6 \%)$ of metabolic alkalosis; of these 13 had a simple disorder while in 68 cases, the metabolic alkalosis was associated to another metabolic or respiratory abnormality. The Stewart method detected metabolic alkalosis in 92 cases $(64.3 \%)$. Of these, 16 were simple and 76 mixed disorders. On 11 undetected metabolic alkalosis by traditional method, 6 cases had low A-tot, 3 cases increased SID and 2 cases both low A tot and incrased SID according to Stewart approach.

Fifteen cases $(10.4 \%)$ of metabolic acidosis were detected by traditional method and twenty-two cases $(15.3 \%)$ by Stewart method. Focalizing on seven discordant cases between the two methods, we found that four had low SID, two increased SIG and one both low SID and increased SIG in agreement to Stewart approach. Results are summarized in table I.

Table I: Metabolic Acid-Base Disorders According to Traditional and Stewart's Method in Investigated Patients.

\begin{tabular}{lcc}
\hline & $\begin{array}{l}\text { Bicarbonate-Based } \\
\text { Method }\end{array}$ & Stewart Method \\
\hline $\begin{array}{l}\text { Total Cases Of Metabolic } \\
\text { Alkalosis }\end{array}$ & 81 & 92 \\
Simple Metabolic Alkalosis & 13 & 16 \\
Mixed Metabolic Alkalosis & 68 & 76 \\
$\begin{array}{l}\text { Total Cases Of Metabolic } \\
\text { Acidosis }\end{array}$ & 15 & 22 \\
Simple Metabolic Acidosis & 3 & 3 \\
Mixed Metabolic Acidosis & 12 & 19 \\
\hline
\end{tabular}

Interestingly, 8 of the 11 cases of metabolic alkalosis and all 7 cases of metabolic acidosis undetected by the traditional method had a mixed disorder.

Statistical analysis performed by chi square test showed no significant difference between two methods to detect both metabolic alkalosis (chi square $=1.443 ; \mathrm{p}=0.226$ ) and acidosis (chi square $=1.118 ; \mathrm{p}=0.290)$.

Linear regression test showed a significant relationship $(r=0.53$; $\mathrm{p}<0.001$ ) between AGcorr and SIG (fig. 1). 


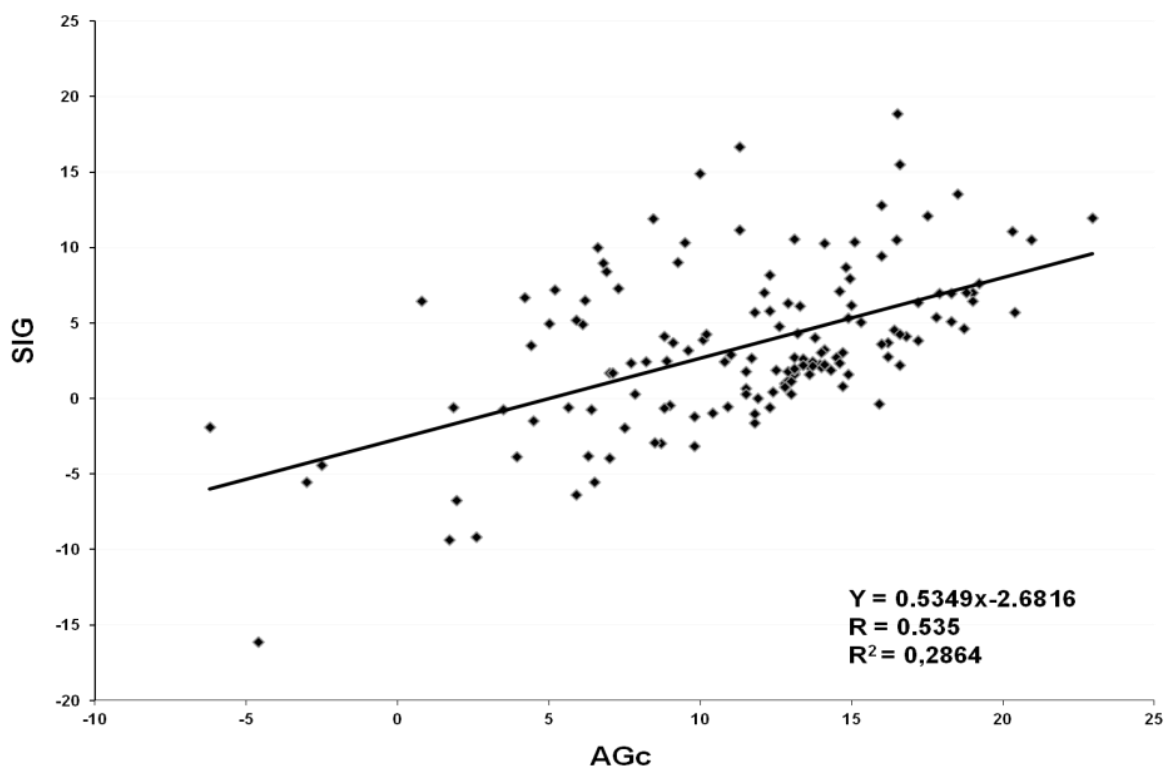

Fig. 1: Relationship between AGcorr and SIG.

\section{Discussion}

The quantitative method proposed by Stewart to assess metabolic acid-base balance was early criticized but in the last years it is increasingly used, especially in critically ill patients while few data are available in internistic clinical setting.

In this pilot study we compared the traditional method, based on Henderson-Hasselbalch equation, and Stewart method in a small cohort of internistic patients.

Many of investigated patients were elderly and had several important comorbidities. Probably for this reason we found a high prevalence of metabolic disorders, especially mixed disorders.

Nevertheless, demographic changes and aging of the general population, observed in the last years, lead us to believe that our patients are likely to be well representative of other internal medicine populations in the same geographical area.

However, in our opinion, these characteristics of our investigated patients did not affect the main aim of our study, i.e. to compare the sensitivity of the two methods, because acid-base status was contemporary assessed in the same patients using both methods Then, the small difference observed in results between the two methods cannot be ascribed to their clinical conditions but to intrinsic differences between the two approaches.

Our results show that bicarbonate-based method failed to detect 11 cases of metabolic alkalosis $(\sim 7 \%)$ and 7 cases of metabolic acidosis $(\sim 5 \%)$ when compared to Stewart method.

Such difference was not statistically significant.

We interestingly found that all 7 cases of acidosis and 8 cases of alkalosis regarded patients with mixed disorders suggesting that the presence of comorbidities can result in a misleading factor even if the expected compensatory rule is applied to the bicarbonate-based approach. For examples, 4 cases are reported in table II.

Patient reported as case 1 in table II, is affected by COPD and heart failure. He shows low $\mathrm{pH}$, hypercapnia and increased bicarbonate ions. These findings, according to bicarbonate-based approach, suggest a compensated chronic respiratory acidosis due to COPD because the increase in bicarbonate ions is consistent with the expected compensatory rule $(0.35 \mathrm{mEq} \mathrm{HCO} 3$ for each $\mathrm{mmHg}$ pCO2 increase; thereby $0.35 \times 37+24=37 \mathrm{mEq} \mathrm{HCO} 3$ expected). According to Stewart approach, this patient also shows a light metabolic alkalosis (increased SID and reduced A-tot) likely due to steroid and furosemide treatment and hypoalbuminemia.
Table II: Examples of Clinical Case

\begin{tabular}{|c|c|c|c|c|}
\hline & Case 1 & Case 2 & Case 3 & Case 4 \\
\hline $\mathrm{pH}$ & 7.30 & 7.45 & 7.27 & 7.46 \\
\hline $\mathrm{PCO}_{2}(\mathrm{mmHg})$ & 77 & 26 & 58 & 30 \\
\hline Alb (g/dl) & 3.4 & 3.7 & 2.8 & 2.7 \\
\hline $\mathrm{P}(\mathrm{i})^{-}(\mathrm{mmol} / \mathrm{L})$ & 2.73 & 1.84 & 0.82 & 0.77 \\
\hline $\mathrm{Na}^{+}(\mathrm{mmol} / \mathrm{L})$ & 145 & 138 & 131 & 134 \\
\hline $\mathrm{K}^{+}(\mathrm{mmol} / \mathrm{L})$ & 5.0 & 3.9 & 4.4 & 4.5 \\
\hline $\mathrm{Ca}(\mathrm{i})^{++}(\mathrm{mmol} / \mathrm{L})$ & 1.11 & 1.15 & 1.4 & 1.6 \\
\hline $\operatorname{Mg}(\mathrm{i})^{++}(\mathrm{mg} / \mathrm{dl})$ & 0.8 & 0.7 & 0.9 & 0.9 \\
\hline $\mathrm{Cl}^{-}(\mathrm{mEq} / \mathrm{L})$ & 103 & 112 & 96 & 94 \\
\hline Lactate $(\mathrm{mmol} / \mathrm{L})$ & 0.5 & 1.1 & 0.7 & 0.7 \\
\hline -HCO3 & 37.9 & 19.8 & 26.6 & 22 \\
\hline AGcorr & 9.4 & 9.7 & 12.5 & 20.75 \\
\hline SIDa & 48.4 & 30.6 & 46 & 48.8 \\
\hline SIG & -1.1 & -1.7 & 11 & 17.66 \\
\hline Atot & 11.73 & 12.57 & 8.73 & 9.14 \\
\hline
\end{tabular}

Patient reported as case 2 is a woman affected by liver cirrhosis and diarrhea. She shows high pH, low pCO2 and low bicarbonate ions. According to traditional method, this patient exhibits a pure compensated chronic respiratory alkalosis, frequently observed in cirrhotics, because decrease of bicarbonate ions is consistent with the expected variation $(0.4 \mathrm{mEq} \mathrm{HCO} 3$ for each $\mathrm{mmHg}$ pCO2 decrease; thereby $24-0.4$ x $14=18.4 \mathrm{mEq} \mathrm{HCO} 3$ expected). In agreement to Stewart method, this patient also shows a light metabolic acidosis (decreased SID) due to diarrhea and minimal metabolic alkalosis (decreased A-tot) due to hypoalbuminemia.

Patient listed as case 3 is a 81 years old man affected by heart failure, diabetes mellitus and acute pneumonia. He shows low $\mathrm{pH}$, increased pCO2 and lightly increased bicarbonate. These findings, in agreement to traditional method suggest an acute respiratory acidosis, consequent to pneumonia, because increase of bicarbonate is consistent with the expected variation $(0.1 \mathrm{mEq} \mathrm{HCO} 3$ for each $\mathrm{mmHg}$ pCO2 increase). The Stewart method also shows a concomitant metabolic alkalosis (increased SID and reduced A tot) and a light metabolic acidosis due to unmeasured anion revealed by increased SIG but not by AGcorr which is substantially in the normal range.

The patient indicated as case 4 is affected by fever and urinary tract infection. He shows an increased pH, low pCO2 and lightly 
reduced bicarbonate concentration. These findings are consistent with acute respiratory alkalosis, in agreement to expected variation $(0.2 \mathrm{mEq} \mathrm{HCO} 3$ for each $\mathrm{mmHg}$ pCO2 decrease), likely due to hyperventilation. The Stewart method also detects a concomitant metabolic alkalosis (increased SID and reduced A-tot) and a metabolic acidosis due to unmeasured anion (increased SIG). In this case the metabolic acidosis is also revealed by increase of AGcorr.

Thus, our results support the hypothesis of a slightly greater reliability of Stewart's method to detect metabolic disorders. Previous studies (Balasubramanyan et al. 1999, Fencl et al. 2000, Martin et al. 2005, Dubin et al. 2007, Boniatti 2009) for assessing the sensitivity of Stewart's approach compared to other methods, conducted on ICU patients, report similar results. Nevertheless, the Stewart method has been criticized. Many clinicians believe that this approach does not improve the ability to diagnose acid-base disorders and it is considered a complex and time-consuming method, unsuitable at the bedside.

In addition, some of underlying principles have been questioned. It has been postulated that SID, pCO2 and A-tot are not really independent of each another because serum chloride, and then SID, could change in response to a primary variation of $\mathrm{pCO} 2$ and plasma albumin by a ${ }^{-} \mathrm{HCO} / \mathrm{Cl}^{-}$exchange across the membrane of red blood cells (Polak et al. 1961, Westen et al. 2003, Langer et al. 2011). This could make us unable to establish whether the change observed in SID is a compensatory effect rather than a concomitant metabolic disorder.

However, it is widely accepted that the metabolic compensation in response to a primitive $\mathrm{CO}_{2}$ alteration is consequent to renal adaptations rather than to the action of hemoglobin $(\mathrm{Hb})$ buffer. Furthermore, results obtained in the experimental in vitro model cannot be fully applied to a more complex in vivo model where it is difficult to carefully assess the water shift across cellular membrane, electrolyte exchanges with the interstitium and electrolyte excretion through the kidney. It has also been reported that the chloride shift from red blood cells also depends upon oxygenation state and hemoglobin concentration by binding/ unbinding of chloride to $\mathrm{Hb}$. It was estimated that only $25-60 \%$ of chloride shift is due to $\mathrm{CO} 2$ variation. In an interesting in vitro in vivo study performed by venovenous extracorporeal membrane oxygenation in critically ill patients, Langer et al. (2011)found that an average $\mathrm{CO} 2$ reduction of about $12 \mathrm{mmHg}$ resulted in an average SID reduction of about $2 \mathrm{mEq}$ only. Thus, the overall effect of $\mathrm{HCO}_{3} / \mathrm{Cl}^{-}$exchange in the red blood cells, consequent to $\mathrm{CO} 2$ variation, on the plasma SID value appears modest and negligible. We also found a significant overall relationship between SIG and AGcorr in the whole study series.

Literature data also report a significant relation between SIG and AGcorr. Surprisingly, we observed two cases of SIG acidosis with normal AGcorr.

The cause of this finding is unclear because both SIG and AG should detect the presence of unmeasured anions.

Importantly, SIG and AGcorr are conceptually similar, because both these parameters represent the amount of abnormal anions present in plasma, but not mathematically.

SIG is obtained subtracting SIDe from SIDa. Then, considering the large number of variables examined for the determination of SIG (SIDa 6 variables - SIDe 3 variables) and the inherent variability (pre-analytical and intra-analytical phase) connected to each of the measurements, SIG may suffer a cumulative error resulting from the sum of those for each of the variables examined. This may affect the reproducibility of the results.

On the other hand, AGcorr does not consider abnormalities in other measured ions as $\mathrm{Mg}^{2+}$ or $\mathrm{Ca}^{2+}$ and the correction for albumin and phosphate is merely an approximation.

Note also that Stewart method does not consider lactate as unmeasured while increase of AGcorr value include serum lactate. In fact, lactate concentration is included in the SIDa calculation; instead AG is calculated only by difference between the main cation and anions $\left(\mathrm{Na}^{+}, \mathrm{Cl}^{-},{ }^{-} \mathrm{HCO} 3\right)$.
Moreover, the $\mathrm{AG}$ varies with $\mathrm{pH}$ and $\mathrm{pCO} 2$ as a response to changing negative charges on albumin and phosphate. The AGcorr has no $\mathrm{pH}$ adjustment while the SIG calculation incorporates $\mathrm{pH}$ and pCO2 in its SIDe component. In an experimental study, Morgan et al. (2007) reported a greater stability of SIG versus AG in response to extreme variations of $\mathrm{pCO} 2$ and $\mathrm{pH}$.

Furthermore, variations of SIG could reflect the presence of unmeasured weak acids resulting by infusion of fluids, gelatins and drugs in hospitalized patients

Then, for all these reasons, the SIG is likely to be a mixture of endogenous and exogenous anions reflecting acid-base imbalance better than AGcorr. This could explain the non-perfect correspondence between the 2 parameters.

\section{Conclusions}

In conclusion, our result showed that in internistic clinical setting bicarbonate-based and Stewart methods are both useful to assess acid-base status in most of hospitalized patients because no significant difference was found and the two approaches are substantially similar in terms of diagnostic performance. Nevertheless, we observed a light disagreement between the two methods suggesting that the traditional method can fail to detect some cases of metabolic acid-base abnormalities. In our experience, this occurred almost exclusively in subjects affected by mixed acid-base disorders despite that expected compensatory rule was applied. In addition, the Stewart method prompts clinicians to consider that $\mathrm{pH}$ is determined not just by the $\mathrm{CO} 2 /$ bicarbonate system but also by other components (strong acids and bases and nonvolatile weak acids and bases) each of that is individually examined leading to a better understanding of pathophysiological alterations

So, although the Stewart method is more complicated and difficult to use at the bedside, it could help clinicians to better assess acidbase balance in some complex patients affected by comorbidities and mixed disorders. Additional studies are required to clarify this matter.

\section{References}

[1] Brønsted JN. [Einige Bemerkungen über den Begriff der Säuren und Basen [Rec Trav Chim Pay-Bas. 1923; 42(8):718-28.

[2] Lowry TM. The uniqueness of hydrogen. Chem Ind. 1923; 42 (3):43-7. https://doi.org/10.1002/jetb.5000420302.

[3] Singer RB, Hastings AB.An improved clinical method for the estimation of disturbances of the acid-base balance of human blood. Medicine. 1948; 27 (2): 223-42. https://doi.org/10.1097/00005792194805000-00003.

[4] Siggaard-Andersen O.The Van Slyke equation. Scand J Clin Lab Invest $\quad$ Suppl. $\quad 1977 ; \quad 146: 15-20$ https://doi.org/10.3109/00365517709098927.

[5] Emmet M, Narins RG. Clinical use of the anion gap. Medicine. 1977; 56 (1):38-54. https://doi.org/10.1097/00005792-19775601000002 .

[6] Gabow PA,Kaehny WD, Fennessey PV, Goodman SI, Gross PA, Schrier RW.Diagnostic importance of an increased anion gap. New England Journal of Medicine. 1980; 303(15):854-8. https://doi.org/10.1056/NEJM198010093031505.

[7] Severinghaus JW.Siggaard-Andersen and the "Great Trans-Atlantic Acid-Base Debate". Scand J Clin Lab Invest Suppl. 1993; 214: 99 104

[8] Stewart PA.How to understand acid-base: A quantitative acid base primer for biology and medicine (1981), New York: Elsevier NorthHolland.

[9] Stewart PA.Modern quantitative acid-base chemistry. Can JPhysiol Pharmacol. 1983; 61(12): 1444-61. https://doi.org/10.1139/y83207.

[10] Guerin C,Nesme P, Leray V, Wallet F, Bourdin G, Bayle F, Germain M, Richard JC. Quantitative analysis of acid-base disorders in patients with chronic respiratory failure in stable or unstable respiratory condition. Respir Care. 2010; 55(11):1453-63.

[11] Fencl V, Jabor A, Kazda A, Figge J. Diagnosis of metabolic acidbase disturbances in critically ill patients. Am J Respir Crit Care Med.2000;162(6):2246-51.

https://doi.org/10.1164/ajrccm.162.6.9904099. 
[12] Boniatti MM. Acid-base disorders evaluation in critically ill patients: we can improve our diagnostic ability. Intensive Care Med.2009; 35 (8): 1377-82. https://doi.org/10.1007/s00134-0091496-2.

[13] Balasubramanyan N, Havens PL, Hoffman GM. Unmeasured anions identified by the Fencl-Stewart method predict mortality better than base excess, anion gap, and lactate in patients in the pediatric intensive care unit. Crit Care Med. 1999; 27 (8):1577-81. https://doi.org/10.1097/00003246-199908000-00030.

[14] Kaplan LJ, Kellum JA.Comparison of acid-base models for prediction of hospital mortality after trauma. Shock. 2008; 29(6):662-6.

[15] Moviat M,van Haren F, van der Hoeven H. Conventional or physicochemical approach in intensive care unit patients with metabolic $\begin{array}{lllll}\text { acidosis. Crit } & \text { Care. 2003; } 7 \text { (3):R41-55 }\end{array}$ https://doi.org/10.1186/cc2184.

[16] Sirker AA,Rhodes A, Grounds RM, Bennett ED. Acid-base physiology: the traditional and the modern approaches. Anaesthesia. 2002; $57(4): 348-56 . \quad$ https://doi.org/10.1046/j.00032409.2001.02447.x.

[17] Kurtz I,Kraut J, Ornekian V, Nguyen MK. Acid-base analysis: a critique of the Stewart and bicarbonate-centered approaches. Am J $\begin{array}{llll}\text { Physiol Renal Physiol.2008; 294(5):F1009-31. } & \text {. }\end{array}$ https://doi.org/10.1152/ajprenal.00475.2007.

[18] Rastegar A. Clinical utility of Stewart's method in diagnosis and management of acid-base disorders. Clin J Am Soc Nephrol. 2009; 4(7):1267-74. https://doi.org/10.2215/CJN.01820309.

[19] Dubin A,Menises MM, Masevicius FD, Moseinco MC, Kutscherauer DO, Ventrice E, Laffaire E, Estenssoro E.Comparison of three different methods of evaluation of metabolic acid-base disorders. Crit Care Med. 2007; 35(5):1264-70. https://doi.org/10.1097/01.CCM.0000259536.11943.90.

[20] Masevicius FD, Dubin A. Has Stewart approach improved our ability to diagnose acid-base disorders in critically ill patients? World $\mathrm{J}$ Crit Care Med 2015; 4(1): 62-70 https://doi.org/10.5492/wjccm.v4.i1.62.

[21] Rastegar A, "Mixed acid-base disorders," Gennari FJ et al. (eds.), Acid Base Disordersand Their Treatment (2005), Boca Ranton: Taylor \& Francis,pp.681-96. https://doi.org/10.1201/b14402-23.

[22] Martin M, Murray J, Berne T, Demetriades D, Belzberg H. Diagnosis of acid-base derangements and mortality predictionin the trauma intensive care unit: The physiochemicalapproach. J Trauma. 2005; 58(2):238-43. https://doi.org/10.1097/01.TA.0000152535.97968.4E

[23] Westen EA, Prange HD. A reexamination of the mechanisms underlying the arteriovenous chloride shift. Physiol Biochem Zool 2003; 76: 603-614 https://doi.org/10.1086/380208.

[24] Langer T, Zani L, Carlesso E, Protti A, Caironi P, Chierichetti M, Caspani ML, Gattinoni L.Contribution of red blood cells to the compensation for hypocapnic alkalosis through plasmatic strong ion difference variations. Critical Care 2011; 15 (Suppl 1): P134 https://doi.org/10.1186/cc9554.

[25] Polak A, Haynie GD, Hays RM, Schwartz WB. Effects of chronic hypercapnia on electrolyte and acid-base equilibrium. I. Adaptation. J Clin Invest1961; 40: 1223-1237 https://doi.org/10.1172/JCI104353.

[26] Morgan TJ, Cowley DM, Weier SL, Venkatesh B. Stability of the strong ion gap versus the anion gap over extremes of PCO2 and $\mathrm{pH}$. Anaesth Intensive Care 2007; 35:370-373. 